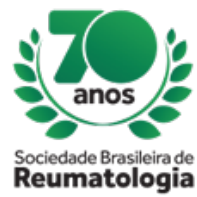

\title{
HENOCH-SCHONLEIN PURPURA AND JUVENILE IDIOPATHIC ARTHRITIS
}

Aline Garcia Islabão (Hospital da Criança de Brasília, BRASILIA, DF, Brasil), Sarah Polyane Silva Veloso (Hospital da Criança de Brasília, BRASILIA, DF, Brasil), Maria Custódia Machado Ribeiro (Hospital da Criança de Brasília, BRASILIA, DF, Brasil), Regiane Duque Minardi Neves (Hospital da Criança de Brasília, BRASILIA, DF, Brasil), Marlon Sousa Lopes (Hospital da Criança de Brasília, BRASILIA, DF, Brasil), Maria Inês Minardi Neves (Hospital da Criança de Brasília, BRASILIA, DF, Brasil)

\section{BACKGROUND}

Henoch-Schonlein purpura (PHS) is the most common leukocytoclastic vasculitis in childhood. It may be associated with other autoimmune or auto-inflammatory diseases such as rheumatic fever, rheumatoid arthritis and familial Mediterranean fever. However, it usually comes isolated and in a single course.

\section{CASE REPORT}

A.K.S.P, at 2 years and 9 months, had palpable purpura in lower limbs and left knee arthritis, diagnosed as PHS. There was early improvement of arthritis. It developed with intense abdominal pain, hematemesis and intestinal bleeding. Initiated prednisolone at a dose for 15 days. She presented a good response to steroid therapy after weaning. After five months she presented right knee arthritis associated with low intensity abdominal pain and low fever for 4 days. Reactivation of purpura, septic arthritis, or reactive arthritis has been suggested. We performed the serological tests for Epstein Baar, toxoplasmosis and cytomegalovirus, which were all negative. Ultrasonography confirmed synovitis, demonstrating a moderate amount of intra-articular fluid. Right knee resonance showed soft tissue edema and small contrast-enhanced effusion. As other causes of chronic arthritis were excluded and it was maintained for 5 months, a diagnosis of juvenile idiopathic arthritis (JIA) was confirmed. Classified as oligoarticular form, rheumatoid factor and antinuclear factor negative and initiated naproxen. She presented good response to treatment with clinical and laboratory remission. The medication was suspended after one year, but patient presented reactivation of arthritis after pneumonia. Carried out infiltration with triamcinolone and restarted anti-inflammatory.

\section{CONCLUSION}

Both clinical conditions presented by the patient may present with joint involvement. PHS may manifest with acute arthritis or arthralgia and is usually oligoarticular. It occurs most often in the fall and winter seasons and is triggers by upper respiratory tract infections. The JIA, however, does not have a seasonal pattern and is subdivided according to the joint involvement and the presence or absence of antibodies.

In the literature, there are 3 adult female patients who presented Henoch-Schönlein purpura and progressed to chronic rheumatoid arthritis. Some studies show mutations of the MEFV gene in JIA related to enthesitis and systemic JIA. These mutations are also found in PHS and could be a relationship between PHS and JIA in the reported patient. We did not find publications on the association of PHS and JIA, emphasizing the unprecedented nature of the case in question. 